\title{
Anxiolytic-like effect of ejaculation upon frustration
}

\author{
ESTEBAN FREIDIN, GISELLE KAMENETZKY, and ALBA ELISABETH MUSTACA \\ Universidad de Buenos Aires-CONICET, Buenos Aires, Argentina
}

\begin{abstract}
In three experiments, we studied the consequences of ejaculation upon the frustrative or contrast response of male rats exposed to reward downshift situations (i.e., surprising changes from $32 \%$ to $4 \%$ sucrose solutions). Similar to what has been found after treatment with anxiolytic agents, consummatory suppression was partially reversed by previous ejaculations in a second postshift trial (Experiments 2 and 3), such a result not having been obtained in a first postshift trial (Experiment 1). Moreover, the effect of ejaculations upon males' behavior during a second postshift trial was transitory, disappearing when assessed during the third and fourth postshift trials (Experiment 3). These results are in accordance with both Amsel's $(1958,1992)$ frustration theory and Flaherty's (1996) multistage hypothesis of successive negative contrast; the diverse factors that are known to modulate contrast effects are considered, including an interpretation of the present data in terms of the anxiolytic-like effect of the ejaculation.
\end{abstract}

When mammals face a downshift in the expected quality or quantity of an appetitive reinforcer (e.g., a $32 \%$-to- $4 \%$ sucrose solution shift), they show a significant decrease in their consummatory performance and an increase in their ambulation and rearing behavior, in comparison with control subjects that have been trained with the lower reinforcer (Flaherty, 1996). This phenomenon has been called the consummatory successive negative contrast (cSNC) effect. According to Flaherty's multistage hypothesis of cSNC (Flaherty, 1996), when an animal detects the occurrence of an unexpected reward downshift, it evaluates the hedonic degree of the new reward by comparing it with the memory of the original reward. If the new reward is valued less than the original one, the animal begins searching for the "missing" reinforcer (Mitchell $\&$ Flaherty, 1998). The processes inherent to this search are thought to occur within the lapse of the first negative contrast session, and none of them is thought to be stressful. The idea of a search for the missing solution has been supported by experiments in which an increase of exploratory behavior (i.e., nose-down locomotion, noseup posture, and open rearing) has been measured when an animal has suffered an incentive downshift from $32 \%$ to

This work was partly supported by UBACYT, Grants P602, N. 1022/03, and CONICET. The authors thank Santiago Pellegrini and an anonymous reviewer for their valuable comments on an earlier version of the manuscript and Mariano Humeniuk for carefully checking the English wording. Finally, the authors thank Charles Flaherty, who died Monday, October 4, 2004, at home. Charlie's work on incentive motivation/reward relativity is one of the best examples of which the authors are aware of a systematic program of research. Correspondence concerning this article should be addressed to E. Freidin or A. E. Mustaca, IIM “A. Lanari," Psicologia Experimental y Aplicada (PSEA), Universidad de Buenos Aires-CONICET, Combatientes de Malvinas 3150, (1426) Buenos Aires, Argentina (e-mail: efreidin@yahoo.com or mustaca@psi.uba.ar).
$4 \%$ sucrose solutions in a radial maze (Pecoraro, Timberlake, \& Tinsley, 1999) and, less directly, by experiments in which it has been found that there is increased locomotion after a 32\%-to- $4 \%$ shift (Flaherty, Blitzer, \& Collier, 1978; Pellegrini \& Mustaca, 2000). Moreover, the absence of stress during the first episode of reward downshift is consistent with data showing that the plasma levels of the stress hormone corticosterone remain unaltered (Flaherty, Becker, \& Pohorecky, 1985; Flaherty, Greenwood, Martin, \& Leszczuk, 1998) and that chlordiazepoxide, diazepam, midazolam, flurazepam, and ethanol are ineffective in reducing behavioral contrast in a first postshift session (Becker, 1986; Becker \& Flaherty, 1982; Flaherty, Grigson, \& Lind, 1990; Flaherty, Clarke, \& Coppotelli, 1996). On the other hand, elevated corticosterone levels after a second postshift day (Mitchell \& Flaherty, 1998) and a partially reversed suppression of consummatory behavior by antianxiety agents have been found in the second negative contrast session (Becker \& Flaherty, 1982; Flaherty et al., 1996). Collectively, these studies suggest that involvement of emotional processes during the first incident of a surprising reward downshift is scarce (if not absent). Stresslike emotional reactions, such as frustration, should be linked to a second stage of the subject's facing a reduction of reward (Flaherty, 1996).

Despite the evidence supporting the multistage hypothesis of cSNC, other authors have stated that the omission or reduction of an expected reinforcer already has aversive effects and elicits negative emotional responses in the first postshift session (Amsel, 1958, 1992; Papini \& Dudley, 1997). For example, Flaherty's (1996) data showing that corticosterone increases after the second postshift day (although not after the first postshift session) could be a byproduct of the time it takes corticosteroids to reach measurable levels, and not a matter of a lack of emotionality 
during that first encounter with the reward downshift (see Papini \& Dudley, 1997). It has been confirmed that the elevation of corticosterone is higher after $20 \mathrm{~min}$ than after 5 min from the start of an instrumental extinction session (Coe, Stanton, \& Levine, 1983). Moreover, Mitchell and Flaherty (1998) have found an anticipatory elevation in plasma corticosterone in shifted animals just before their placement in the apparatus on the second postshift day. Besides the hypothesis that emotional processes are already involved during the first occurrence of reward reduction, the lack of effects of anxiolytics upon an animal's performance in the first postshift day could be explained by considering that antianxiety drugs do not usually affect an animal's reaction to an unconditional aversive stimulus but actually exert their effect upon subjects facing stimuli that anticipate a forthcoming aversive situation (Gray, 1985). For example, a review of over 400 studies in which many different species and antianxiety drugs were used showed that four kinds of inputs (including signals of punishment, innate fear stimuli, and signals of reward omission) initially produce behavioral inhibition, after which they produce increased attention and increased arousal (Gray, 1982). Interestingly, antianxiety drugs block these three behavioral outputs (see Gray, 1985).

In agreement with this point of view, Amsel (1958, 1992) stated, in his frustration theory, that a surprising reward omission induces an internal state known as primary frustration, which has both behavioral and physiological correlates and is characterized by its unconditioned aversion and its strengthening properties (i.e., invigoration of subsequent responses). Some authors have interpreted the increased locomotive activity following a reward downshift as an index of emotionality. Indeed, Flaherty et al. (1978) found that $32 \%$-to- $4 \%$ shifted animals exhibited significant increases in activity, although they were less likely to sample from new drinking tubes than were nonshifted controls, from which it could be inferred that the increased locomotion was not due to increased exploration.

Amsel (1958) also states that primary frustration as an aversive internal state could be associated with either discrete or contextual cues, which then give rise to conditioned frustration. For example, rats learn to escape from a stimulus associated with the omission of an expected reward just as they learn to escape from a key paired with electric shocks (Daly, 1974). Indeed, the contrast effect on the second postshift day is more related to conditioned fear, as has been revealed in a factor-analytic study (Flaherty et al., 1998). Moreover, first and second trials of a consummatory extinction procedure differed in their consequences upon the sexual performances of male rats. After a first reward omission trial, experimental subjects have been shown to perform more mounts without intromission, to have lower copulatory efficiencies, and to exhibit a trend toward shorter mount latencies and shorter intermount intervals than did control subjects, but they presented a delayed ejaculatory response after a second postshift trial (Freidin \& Mustaca, 2004). These results could be interpreted in terms of the effects of primary and conditioned frustration, respectively, upon sexual behav- ior (Amsel, 1958; for a review of the consequences of surprising reward omissions, see Papini \& Dudley, 1997).

In short, both Flaherty's (1996) multistage hypothesis of cSNC and Amsel's (1958) frustration theory are consistent with the idea that two distinct behavioral/physiological sequential processes take place in animals facing a reward downshift, despite their agreement or disagreement with respect to the involvement of emotional processes in the first encounter with the reward reduction.

In another line of research, Fernández-Guasti, RoldánRoldán, and Saldívar (1989) showed a reduction in the burying behavior of male rats after their ejaculation. Defensive burying is an innate response exhibited by rats toward aversive stimuli, which consists in burying welllocalized sources of noxious stimulation. Several authors have shown that anxiolytics suppress defensive burying, which suggests that this paradigm can serve as a behavioral model for the study of anxiety in animals (see RodríguezManzo, López-Rubalcava, \& Fernández-Guasti, 1999). It has been reported that the time that a rat spent spraying and pushing bedding material (i.e., burying behavior) toward a conspicuous source of noxious stimulation (an electric prod emerging from one of the walls of the experimental chamber) was significantly decreased when the animal had just had an ejaculation, when compared with either controls unexposed to females or animals allowed to reach just five intromissions (Fernández-Guasti et al., 1989). As far as our knowledge goes, the defensive burying test has been the only procedure reported in the literature in which anxiolytic-like effects of ejaculation have been proven.

In the present experiments, we tested the potential effect of ejaculation upon the goal-tracking time, ambulation, and rearing behavior of rats subjected to a cSNC. This study was conducted with many theoretical and methodological goals in mind: first, to test the idea that two distinct sequential processes take place in a reward downshift situation, which predicts different consequences of ejaculations in the first and the second postshift trials of a cSNC (Amsel, 1992; Flaherty, 1996); second, to extend ejaculation effects to a stressful situation without the presentation of nociceptive stimuli; and finally, to generalize the anxiolytic power of ejaculation with a different stress procedure.

In our standard cSNC procedure, during the preshift phase, one group of animals (i.e., Group 32-4) received two daily 5 -min periods of access to a $32 \%$ sucrose solution in conditioning boxes for 6 days (i.e., 12 preshift trials), whereas the control group received the same two daily 5 -min trials, but with access to a less preferred $4 \%$ sucrose solution (i.e., Group 4-4). During the postshift phase, the less preferred $4 \%$ sucrose solution was presented to all the subjects in their 5-min trials. In Experiment 1 , we analyzed the effect of two previous ejaculations upon the behavior of the animals during the first postshift trial; in the second experiment, we assessed the effect of previous ejaculations on the behavior of males during the second postshift trial; and in Experiment 3, we replicated the effect of ejaculations on the behavior of 
males during the second postshift trial, and we evaluated the duration of this ejaculatory effect, as it disappeared during the course of subsequent postshift trials.

\section{EXPERIMENT 1}

The goal of the present experiment was to evaluate the consequences of ejaculations - one approximately $20 \mathrm{~h}$ before the test and the other right before it - upon the behavior of rats in the first postshift trial of a $32 \%$-to- $4 \%$ reward downshift situation. The behavior of downshifted ejaculators was compared with that of ejaculators that did not experience the reward downshift and with that of animals that experienced the downshift but had not been allowed to copulate with receptive females.

\section{Method}

Subjects. The subjects were 41 ejaculators selected out of 53 adult Wistar male rats (the sexual pretest are described in the Procedure section); all were experimentally naive and were bred at the Instituto de Investigaciones Médicas Alfredo Lanari, Universidad de Buenos Aires. The rats weighed between 220 and $352 \mathrm{~g}$. They were housed in individual wire cages and were exposed to a 12:12-h light:dark cycle (lights on from 06:00 to 18:00 h). The temperature was maintained at $23^{\circ} \mathrm{C}$. The animals had ad lib access to water throughout the experiment. The rats were deprived of food until they reached $90 \%$ of their free-feeding weight and were maintained at this level during the course of the experiment.

Nineteen ovariectomized female Wistar rats were housed in groups of 4 animals. They were used both in the males' sexual pretests and to induce ejaculatory responses of animals in the ejaculatory condition.

Apparatus. The metallic home cages of the male rats were $28 \mathrm{~cm}$ wide, $26 \mathrm{~cm}$ long, and $23 \mathrm{~cm}$ high, with bars $0.1 \mathrm{~cm}$ in diameter, separated by $1.5 \mathrm{~cm}$. Consummatory training occurred in four conditioning boxes (MED Associates), each $29.2 \mathrm{~cm}$ long, $24.1 \mathrm{~cm}$ wide, and $21 \mathrm{~cm}$ high. The floor was made of aluminum bars measuring $0.4 \mathrm{~cm}$ in diameter and spaced $1.1 \mathrm{~cm}$ apart (from center to center). On one of the lateral walls, there was a cubicle measuring $5 \mathrm{~cm}$ in width, $5 \mathrm{~cm}$ in height, and $3.5 \mathrm{~cm}$ in depth, located $10 \mathrm{~cm}$ above the floor. The sipper tube was inserted into it from outside the box, protruding approximately $2 \mathrm{~cm}$ inside the cubicle. The rats had to insert their heads into this cubicle to reach the sipper tube, from which they could drink either a $32 \%$ sucrose solution $(32 \mathrm{~g}$ of commercial sugar for each $68 \mathrm{ml}$ of tap water) or a $4 \%$ sucrose solution (4 $\mathrm{g}$ of sugar for each $96 \mathrm{ml}$ of water). Goal-tracking time (in 0.01 -sec units) was measured by a computer that registered the amount of time that a photocell located in front of the drinking tube was activated during a trial. Each box was enclosed in a sound- and light-attenuating cubicle equipped with a source of white noise and diffuse houselight.

All the subjects were videorecorded during the postshift trial, using two video cameras (Sharp VL-E685U and Sony Digital 8 DCR-TRV310 NTSC).

Procedure. The procedure had the following sequence.

Sexual pretests. Males were pretested for masculine sexual behavior. Each male was taken to the experimental room in its home cage, and approximately 2 min later, an ovariectomized receptive female was placed in the cage of the male rat. Males displaying ejaculation at least once in a maximum of five 15 -min sessions were selected. The female rats were brought into heat by administration of estradiol benzoate $(50 \mu \mathrm{g} \mathrm{EB} / 0.1 \mathrm{ml}$ olive oil, $48 \mathrm{~h}$ before testing) and progesterone ( $500 \mu \mathrm{g} \mathrm{P} / 0.1 \mathrm{ml}$ olive oil, $3 \mathrm{~h}$ before testing).

Preshift phase. Pairs of rats matched for weight were randomly assigned to one of two groups: Group 32-4 (exposed to the 32\% sucrose solution during the preshift phase and to the $4 \%$ solution during the postshift trial; $n=23$ ) or Group 4-4 (exposed to the 4\% sucrose solution during both the preshift and the postshift trials; $n=$ 18). The day before the beginning of the experiment, all the subjects received access to the training solution in their home cages $(32 \%$ or $4 \%$, depending on group assignment). A drinking bottle with $20 \mathrm{ml}$ of solution was placed in the cages for $20 \mathrm{~min}$. The preshift phase started the following day and lasted for 12 trials, administered at a rate of 2 trials per day. Within a day, the intertrial interval was 2-2.5 $\mathrm{h}$; between days, the intersession interval was 20-21 $\mathrm{h}$. The rats were placed in their home cages during both intervals. Squads of 4 rats were trained simultaneously. The order of the squads and the conditioning box assigned to each animal were rotated across trials. Preshift trials consisted of placing the males in the conditioning boxes, where they had access to either the $32 \%$ sucrose solution (Group 32-4) or the 4\% sucrose solution (Group 4-4) by licking the sipper tube.

Postshift phase. After the end of the preshift phase, pairs of rats within each group matched for preshift goal-tracking time were randomly assigned to one of two conditions: the ejaculatory condition (rats allowed to ejaculate approximately $20 \mathrm{~h}$ before and immediately before the postshift trial; Group Ej32-4, $n=9$, and Group $\mathrm{Ej} 4-4, n=9$ ) and the nonejaculatory condition (rats not exposed to females; Group 32-4, $n=14$, and Group 4-4, $n=9$ ). The subjects assigned to the ejaculatory condition were taken to the experimental room and were exposed, in their home cages, to a receptive female $20 \mathrm{~h}$ before the postshift trial. After the male reached its first ejaculation, the female was retired from the cage. On the next day, immediately before the postshift trial, the ejaculators were exposed again to a receptive female. In this case, once the males had reached ejaculation, they remained in their home cages for about a minute and then were placed in the conditioning boxes for the postshift trial. In experiments that have tested the anxiolytic-like effect of ejaculation upon the burying behavior of rats (Rodríguez-Manzo et al., 1999), it has been shown that the highest anxiolytic effect occurred after two ejaculations, $24 \mathrm{~h}$ before and right before the burying test. Hence, we chose to conduct a similar two-ejaculations procedure in the present experiment, in order to maximize the independent variable.

Finally, the postshift trials were identical to the preshift trials in all aspects, with the exception that all the subjects received the $4 \%$ solution and the males' behavior was videorecorded during those $5 \mathrm{~min}$.

Goal-tracking time was measured as the dependent measure in all the preshift and postshift trials. Previous studies have shown that goal-tracking time is positively correlated with the volume of reinforcer consumed by animals (Mustaca, Freidin, \& Papini, 2002; Mustaca \& Martelli, 2000). Ambulation and rearing behavior were measured from the video recording only for the postshift trial. Two observers watched the videos without knowing the animal group assignment and registered, every $10 \mathrm{sec}$, whether the animal was ambulating, rearing, or not doing either of these. Interobserver reliability was rated above $91 \%$ (i.e., agreements/total observations).

Two-way ANOVAs (i.e., reward shift $\times$ ejaculatory condition) were performed on goal-tracking time, ambulation, and rearing behavior. The trials factor was included as a repeated measure for the analysis of the goal-tracking time data. Bonferroni tests were conducted for pairwise comparisons of Group 32-4 and Group Ej32-4 and of Group 4-4 and Group Ej4-4. The alpha value was set at the .05 level, and Bonferroni adjustments were conducted for pairwise comparisons $(\alpha c=.0125)$.

\section{Results and Discussion}

The main preshift and postshift consummatory training results are presented in Figure 1.

Preshift phase. The average goal-tracking time grew throughout the preshift phase for all the groups, although the animals that received a $32 \%$ solution presented a higher performance than did those that received the $4 \%$ solution. 


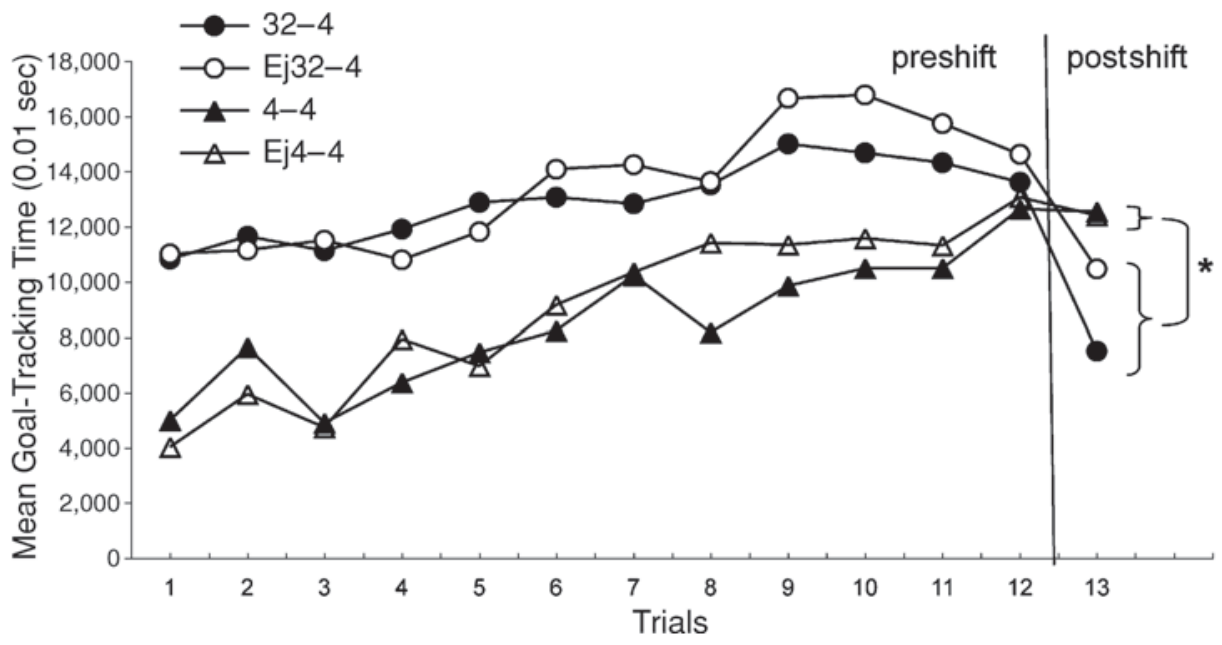

Figure 1. Experiment 1: consummatory performance, measured in terms of goal-tracking time, as a function of training phase, reward shift, and ejaculatory condition. Groups 32-4 and Ej32-4 received $32 \%$ sucrose solution in the preshift trials and $4 \%$ solution in the postshift trial; Groups 4-4 and Ej4-4 received $4 \%$ solution in both the preshift and the postshift trials. Only the rats in Groups Ej32-4 and Ej4-4 ejaculated $20 \mathrm{~h}$ before and immediately before the postshift trial. $\quad * p<.05$.

This difference in the consummatory behavior between groups was supported by a statistical analysis of the 12 preshift trials, which showed significant effects of both group $[F(1,37)=19.96, p<.01]$ and trials $[F(11,407)=$ $15.48, p<.01]$; on the other hand, the reward shift $\times$ trials interaction was not significant $[F(11,407)=1.68$, n.s. $]$. As was expected, given that it was implemented once the preshift phase was over, the ejaculatory condition showed no significant main effects interactions (all $F_{\mathbf{s}}<1$ ).

Postshift phase. The $32 \%$-to- $4 \%$ shift resulted in a sharp decline in goal-tracking time for both Group 32-4 and Group Ej32-4, whereas the groups that remained with the same reinforcer (i.e., Group 4-4, and Group Ej4-4) presented a stable performance between the preshift and the postshift trials. An analysis of the last preshift trial and the postshift trial together indicated a significant reward shift $\times$ trials interaction $[F(1,37)=7.17, p<.01]$. The ejaculatory condition factor did not present either a significant main effect $[F(1,37)=1.07]$ or a significant interaction with any of the other factors (all $F_{\mathrm{s}}<1$ ). An analysis of the postshift trial alone indicated a significant effect of reward shift $[F(1,37)=6.29, p=.01]$, whereas the effect of ejaculatory condition $[F(1,37)=1.02]$ and the interaction of reward shift and ejaculatory condition $[F(1,37)=1.24]$ were not significant.

Despite the fact that the postshift performance of the animals belonging to Group Ej32-4 appears to be higher than that of the subjects in Group 32-4, as can be seen in Figure 1, Bonferroni's planned pairwise comparisons between those groups resulted in a nonsignificant difference between them $(p>.02)$. The same result was found when the postshift goal-tracking times for Groups 4-4 and $\mathrm{Ej} 4-4$ were compared $(p>.02)$.

Mean proportions of ambulation and rearing behavior for each of the four groups during postshift are shown in Figure 2.
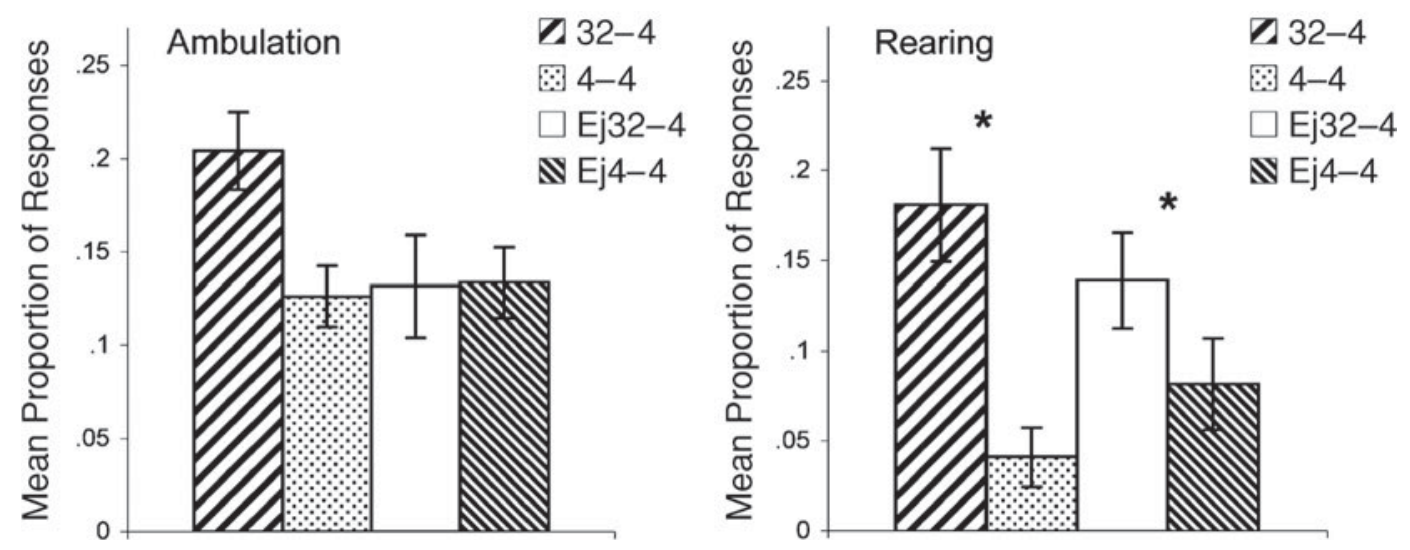

Figure 2. Experiment 1: ambulation and rearing behavior as a function of reward shift and ejaculatory condition. Error bars denote 1 SEM. $\quad * p<.05$. 
Ambulation did not significantly differ between groups, as is shown by the statistical analysis [reward shift, $F(1,37)=$ 2.25 , n.s.; ejaculatory condition, $F(1,37)=1.56$, n.s.; reward shift $\times$ ejaculatory condition, $F(1,37)=2.51$, n.s.]. Also, Bonferroni's pairwise comparisons showed a lack of substantial differences between Group 32-4 and Group Ej32-4 and between Group 4-4 and Group Ej4-4 (all $p \mathrm{~s}>.02)$. Results obtained after percentages of rearing behavior were analyzed were as follows: Animals that experienced the reward downshift (averages for Groups 32-4 and Ej32-4: 18\% and 14\%, respectively) presented a higher percentage of rearing behavior than did the subjects of the nonshifted condition (averages for Groups 4-4 and Ej4-4: 4\% and 8\%, respectively), a difference that proved statistically reliable [reward shift, $F(1,37)=$ $12.94, p<.01]$. As for the previous analysis, there was no significant effect of ejaculatory condition [ejaculatory condition, $F(1,37)=0.02$, n.s.; reward shift $\times$ ejaculatory condition, $F(1,37)=2.50$, n.s.].

The present results show that all the 32\%-to- $4 \%$ downshifted animals presented similar goal-tracking times, percentages of ambulations, and percentages of rearing behavior, independently of having or not having ejaculated before the test trial. In addition, the shifted subjects, whether or not they were ejaculators, showed significantly lower consummatory behavior and significantly higher percentages of rearing behavior, in comparison with the nonshifted animals, during the postshift trial. These two findings suggest that the contrast effect, usually seen after a reward downshift, is not affected by previous ejaculations when assessed in the first postshift trial. Moreover, from the present results, it also follows that the behavior of the control subjects which always drank the $4 \%$ solution, was not altered by ejaculations either.

\section{EXPERIMENT 2}

Flaherty et al. (1985; Flaherty et al., 1998) found increased corticosterone levels after the second postshift trial of a cSNC procedure, but not after the animals first detected the shift. They also observed that anxiolytic agents (e.g., chlordiazepoxide) reduced the behavioral contrast effect on the second trial after the reinforcement downshift, but not on the first contrast trial (Becker \& Flaherty, 1982; Flaherty, Grigson, \& Lind, 1990; Flaherty et al., 1996). On that basis, different authors have elaborated theories about frustration phenomena in which two distinct behavioral/physiological sequential processes taking place in the animals that face a reward downshift are considered (Amsel, 1992; Flaherty, 1996).

If we consider that the first and the second instances of reward reduction lead to different consequences, as Flaherty's (1996) multistage hypothesis proposes and Amsel's (1992) frustration theory predicts, we may expect ejaculations prior to the second postshift trial to have a different effect on cSNC, in comparison with ejaculations prior to the first postshift trial. More specifically, if an anxiolytic-like effect of ejaculation (Fernández-Guasti et al., 1989; Rodríguez-Manzo et al., 1999) is expected, ejaculators will be predicted to behave like those subjects treated with anxiolytic drugs in cSNC situations in previous studies (Becker \& Flaherty, 1982; Flaherty, Grigson, \& Lind, 1990; Flaherty et al., 1996). Therefore, we expected to find that ejaculations before the second postshift trial would increase the consummatory recovery and decrease the subjects' ambulation and rearing behavior in a $32 \%$-to- $4 \%$ downshift situation.

The goal of the present experiment was to evaluate the consequences of ejaculations for the behavior of rats in the second postshift trial of a 32\%-to-4\% reward downshift situation. The behavior of downshifted ejaculators was compared with that of animals that experienced the reward downshift but were not allowed to copulate. Controls that always consumed the $4 \%$ solution were omitted in the present experiment, because the data from Experiment 1 and from other experiments performed in our laboratory (unpublished data) showed that ejaculations seemed not to affect the performance of nonshifted subjects on any of the dependent measures under consideration.

\section{Method}

Subjects and Apparatus. The subjects were 20 ejaculators selected out of 25 adult Wistar rats, all experimentally naive, bred and maintained under the same environmental and food deprivation conditions as those described in the previous experiment. The rats weighed between 220 and $296 \mathrm{~g}$. The 19 ovariectomized female Wistar rats in Experiment 1 were also used in the males' sexual pretests and in the stimulation of ejaculatory responses of animals in the ejaculatory condition. The home cages of the males and the conditioning boxes were the same as those in Experiment 1.

Procedure. The procedure was identical to that described in the previous experiment, with the following exceptions. (1) Only two groups were trained. After the end of the preshift phase, pairs of rats matched for preshift goal-tracking time were randomly assigned to one of two conditions: the ejaculatory condition (rats allowed to ejaculate both approximately $20 \mathrm{~h}$ before and immediately before the second postshift trial; Group Ej32-4, $n=11$ ) and the nonejaculatory condition (rats not exposed to females; Group 32-4, $n=8$ ). (2) Two postshift trials were conducted. (3) Ambulation and rearing behavior were measured in the second postshift trial.

An ANOVA for two independent groups (32-4 and Ej32-4) was conducted for goal-tracking time, ambulation, and rearing behavior. The trials factor was included as a repeated measure for the analysis of goal-tracking time data. The alpha value was set at the .05 level.

\section{Results and Discussion}

A rat from Group Ej32-4 was eliminated from the study because it failed to reach the ejaculatory response before the second postshift trial, and another animal was eliminated because it became ill during the preshift phase. Therefore, all the statistical analysis were conducted with the scores of 18 subjects (i.e., Group Ej32-4, $n=10$, and Group 32-4, $n=8$ ).

The main preshift and postshift consummatory training results are presented in Figure 3.

Preshift phase. Throughout this phase, the growth of the average goal-tracking time was similar for both groups, which is supported by the statistical analysis of the 12 preshift trials [trials, $F(11,176)=16.58, p<.01$; ejaculatory condition and ejaculatory condition $\times$ trials, $\left.F_{\mathrm{s}}<1\right]$. 


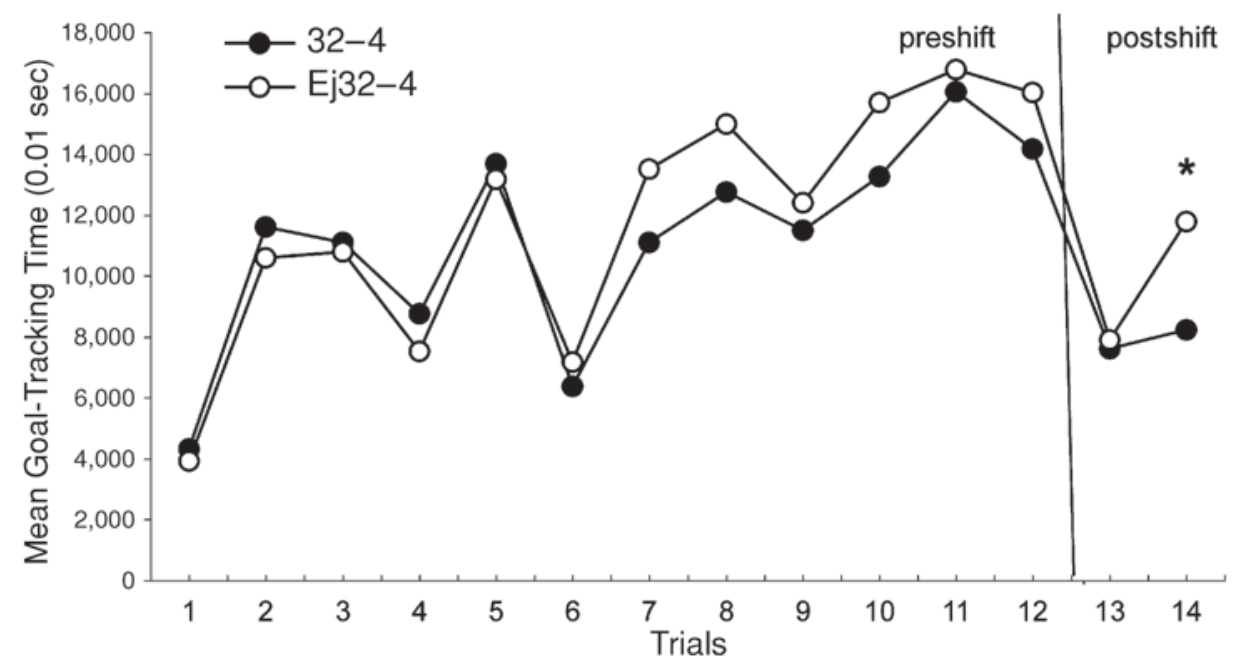

Figure 3. Experiment 2: consummatory performance, measured in terms of goal-tracking time, as a function of training phase, and ejaculatory condition. Groups 32-4 and Ej32-4 received 32\% sucrose solution in preshift trials and $4 \%$ solution in postshift trials, but only the rats in the Group Ej32-4 ejaculated $20 \mathrm{~h}$ before and immediately before the second postshift trial. ${ }^{*} p<.05$.

Postshift phase. The $32 \%$-to- $4 \%$ shift resulted in a sharp decline in goal-tracking time for the animals in both groups. An analysis of the consummatory performance during the last preshift and first postshift trials, taken together, indicated a significant effect of trials $[F(1,16)=43.86, p<.01]$, with no significant effect of ejaculatory condition and no ejaculatory condition $\times$ trials interaction (both $F \mathrm{~s}<1$ ). Interestingly, as can be seen in Figure 3, after achieving the ejaculations, the animals in Group Ej32-4 presented a higher goal-tracking time recovery in the second postshift trial, in relation to the animals in Group 32-4, which is supported by the analysis of the consummatory behavior of the second postshift trial [ejaculatory condition, $F(1,16)=5.26, p<.03$ ]

Mean proportions of ambulation and rearing behavior for each group during the second postshift trial are shown in Figure 4.

The animals from the Group 32-4 dedicated a significantly higher proportion of responses to ambulation (av- erage, $27 \%$ ) than did the subjects in Group Ej32-4 [average, $17.5 \% ; F(1,16)=6.12, p<.02]$; groups, however, did not differ in the proportion of responses dedicated to rearing behavior $[F(1,16)=3.1478, p=.09]$.

The present results indicate that previous ejaculations affected the behavior of the subjects on the second postshift trial of the cSNC procedure. Specifically, ejaculators presented an increased consummatory recovery from the contrast effect, when compared with downshifted subjects that did not ejaculate. Moreover, the animals that ejaculated spent less time ambulating than did the nonejaculators.

\section{EXPERIMENT 3}

It has been reported that anxiolytic agents facilitate recovery from consummatory contrast, as measured during the second postshift trial (Becker \& Flaherty, 1982; Flaherty et al., 1996). Besides, Flaherty, Grigson, and Rowan (1986) and Flaherty, Grigson, et al. (1990) have
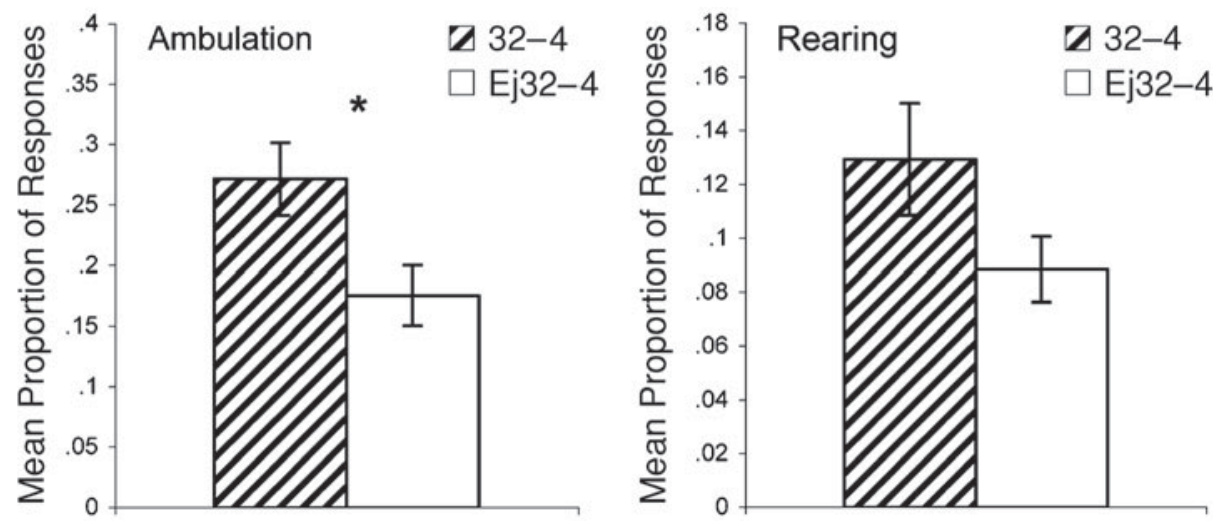

Figure 4. Experiment 2: ambulation and rearing behavior as a function of ejaculatory condition. Error bars denote 1 SEM. $*$ * $p<.05$. 


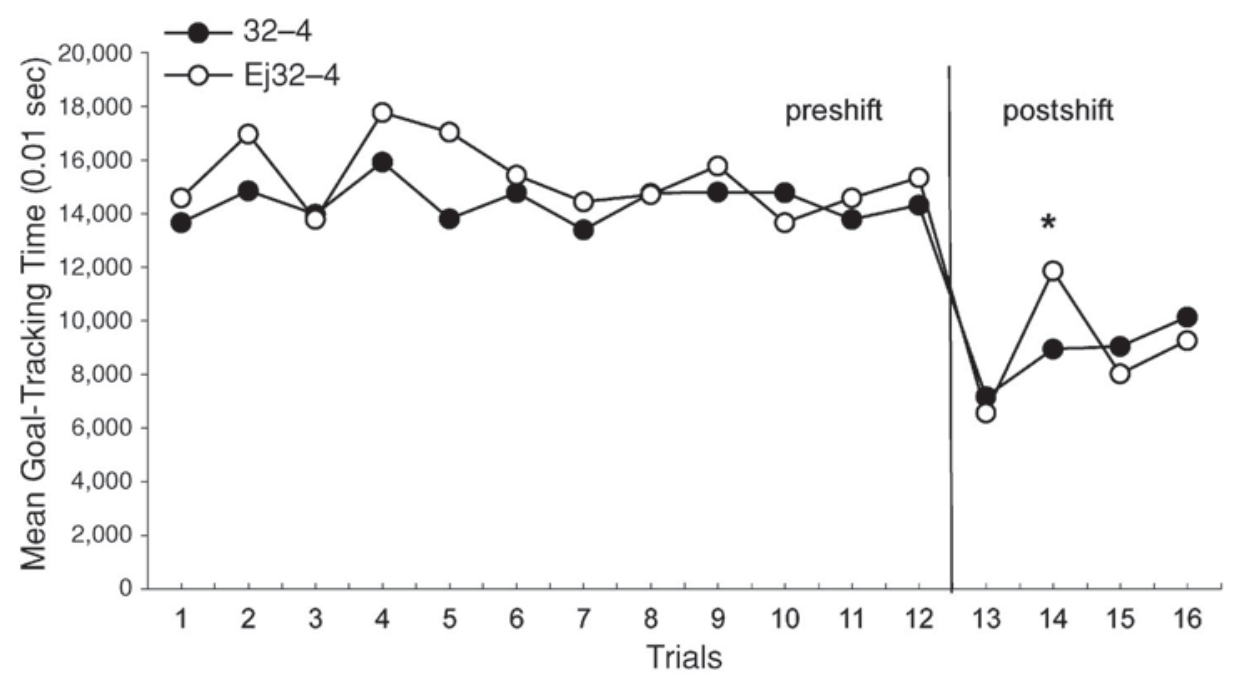

Figure 5. Experiment 3: consummatory performance, measured in terms of goal-tracking time, as a function of training phase and ejaculatory condition. Groups 32-4 and Ej32-4 received 32\% sucrose solution in preshift trials and $4 \%$ solution in postshift trials, but only the rats in Group Ej32-4 ejaculated $20 \mathrm{~h}$ before and immediately before the second postshift trial. $* p<.05$.

shown that the administration of chlordiazepoxide and midazolam, respectively, prior to to the second postshift session in a cSNC, has an acute consummatory recovery effect only during that session, given that the animals presented a behavioral contrast again in the third and fourth postshift sessions.

The goal of this third experiment was twofold: first, to replicate the effect of ejaculations upon the animals' behavior during the second postshift trial of a $32 \%$-to- $4 \%$ downshift situation; second, to test the duration of this effect of ejaculations prior to the second postshift trial, up to the time that it disappeared during subsequent postshift trials. If the effect of ejaculations is acute, we would expect ejaculators and nonejaculators to differ in their behavior during the second postshift trial, but not during the subsequent postshift trials. According to this prediction, males should present higher consummatory performances and lower percentages of ambulations and rearing behavior right after the ejaculatory response (i.e., during the second postshift trial), relative to control subjects, and then those differences between groups should disappear in subsequent postshift trials.

\section{Method}

Subjects, Apparatus, and Procedure. The subjects were 16 adult Wistar rats, selected through a sexual pretest similar to those used in the previous experiments. All these ejaculators had previous experience drinking the $4 \%$ sucrose solution in the conditioning boxes, but they did not participate in Experiment 1 or 2. The animals were bred and maintained under the same environmental and food deprivation conditions as those described for previous experiments. The rats weighed between 225 and $300 \mathrm{~g}$. Also, the same female rats as those in Experiments 1 and 2 were used. The home cages of the males, the conditioning boxes, and the video cameras were the same as those described previously.

The procedure, dependent measures, and statistical data analysis were identical to those described in Experiment 2, with the only exception being that the postshift phase lasted for four trials, instead of two. After the end of the preshift phase, pairs of rats matched for preshift goal-tracking time were randomly assigned to one of two conditions: Group 32-4 $(n=8)$ and Group Ej32-4 $(n=8)$. The animals from Group Ej32-4 were allowed to ejaculate $20 \mathrm{~h}$ before and immediately before the second postshift trial, whereas the subjects from Group 32-4 had no access to females.

\section{Results and Discussion}

The main preshift and postshift consummatory training results are presented in Figure 5.

Preshift and postshift phases. Unlike the results in the previous experiments, the animals in both groups did not show a consummatory learning curve during the preshift phase, probably because they had already had extensive experience with sucrose solutions. Nevertheless, the important outcome for the goals of the experiment was that the goal-tracking time for Groups 32-4 and Ej32-4 did not statistically differ, either at the end of the preshift phase or in the first postshift trial (both $F_{\mathrm{s}}<1$ ).

An analysis of the consummatory performance in the second postshift trial indicated a significant effect of ejaculatory condition $[F(1,14)=6.84, p=.02]$, which meant that after achieving the ejaculations, the animals in Group Ej32-4 showed a higher goal-tracking time recovery on that postshift trial, in relation to the animals in Group 32-4.

As can be seen in Figure 5, goal-tracking time differences between groups - seen on the second postshift trialdisappeared on Postshift Trials $3[F(1,14)=1.41$, n.s.] and $4(F<1)$.

Mean proportions of ambulation and rearing behavior for each group during the second postshift trial are shown in Figure 6.

Males from Group 32-4 spent a significantly higher proportion of trial time in rearing behavior (average, 
$17 \%)$ than did the subjects in Group Ej32-4 [average, $8.5 \% ; F(1,14)=5.35, p<.04]$, and no differences between groups appeared in the proportion of ambulations $(F<1)$.

On one hand, the results of Experiment 3 replicated the finding in Experiment 2, in which it was shown that previous ejaculations facilitated the consummatory recovery during the second postshift trial of a $32 \%$-to- $4 \%$ reward downshift situation. Moreover, in the present experiment, it was also suggested that the ejaculation effect upon consummatory behavior was transient - restricted only to the second postshift trial — given that group differences in goal-tracking time were lost on the third and the fourth postshift trials. The finding of a transitory aspect of the ejaculation effect on frustration, though, cannot be taken to be conclusive, because all the subjects had had previous experience with the $4 \%$ reward. Thus, the rats actually experienced two surprising reward changes (i.e., one from $4 \%$ to $32 \%$, and the other from $32 \%$ to $4 \%$ ), which might have interacted with the ejaculation effect. However, as can be seen from a comparison of Figures 3 and 5, the naive male rats in Experiment 2 and the 4\% experienced animals in Experiment 3 presented remarkably similar ejaculation effects upon goal-tracking time in the second postshift trial, which suggests that the previous experience of the rats in the last experiment may not be relevant for the present analysis.

On the other hand, although the animals that ejaculated spent less time rearing than did the nonejaculators, Experiment 2's ambulation differences between groups were not replicated in Experiment 3. This incongruity between experiments could be interpreted by considering ambulation and rearing behavior as two exploratory responses, both part of a search repertoire evoked by incentive downshifts (Pecoraro et al., 1999). Therefore, Experiments 2 and 3 showed that these exploratory behaviors evoked by the reward downshift could be reduced by previous ejaculations.

\section{GENERAL DISCUSSION}

In previous studies, it has been found that male rats present a reduction in innate stress responses, such as defensive burying behavior, after having an ejaculation (FernándezGuasti et al., 1989; Saldívar, Ríos, \& Fernández-Guasti, 1991). Moreover, the reduction in burying behavior was more abrupt in male rats that had had an ejaculation $24 \mathrm{~h}$ previous to the burying test and an ejaculation immediately before the test session than in males that had had a single ejaculation right before the test (Rodríguez-Manzo et al., 1999). This ejaculatory effect on burying behavior has been interpreted as anxiolytic-like, because it produces a reduction in the amount of a response known to be sensitive to drugs with anxiolytic properties (Treit, Pinel, $\&$ Fibiger, 1981).

On the other hand, it has been suggested that when a mammal faces a downshift in the expected quality or quantity of an appetitive reinforcer, it has an anxiety-like frustrative response (Amsel, 1958, 1992; Papini \& Dudley, 1997), evidenced in elevated plasma levels of the stress hormone corticosterone (Flaherty et al., 1985). Moreover, drugs classified as anxiolytics in humans tend to reduce the behavioral effects of unexpected downward shifts of reward, such as the consummatory suppression of the postshift reinforcer and the augmented ambulatory and rearing behaviors (Gray, 1977, 1982; Mustaca, Bentosela, \& Papini, 2000).

One of the main goals of this study was to assess the effect of ejaculation upon the behavior of male rats faced with a $32 \%$-to- $4 \%$ reward downshift. In Experiment 1, shifted animals expressed a consummatory contrast, aside from having or not having ejaculated before the first postshift trial. This result is in agreement with previous findings in which anxiolytic agents did not affect the behavior of animals during the first postshift trial but, rather, reduced the consummatory suppression during the second postshift trial in a cSNC situation (Becker \& Flaherty, 1982; Flaherty, Grigson, et al., 1990; Flaherty, Grigson, \& Lind, 1990; Flaherty et al., 1996). Thus, we planned to test the ejaculatory effect on the second postshift trial (Experiment 2). The data in Experiment 2 indicates that the rats facing the downward shift presented an incremented consummatory recovery and a reduced ambulatory behavior during the second postshift trial only when they had had previous ejaculations. In Experiment 3, the facilitatory effect of ejaculations upon consummatory be-
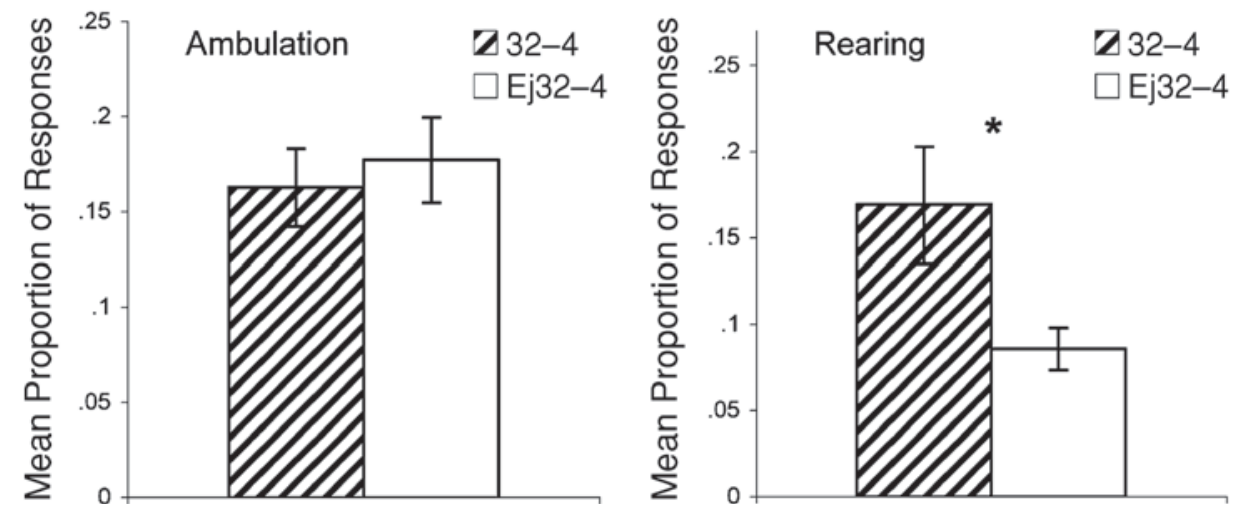

Figure 6. Experiment 3: ambulation and rearing behavior as a function of ejaculatory condition. Error bars denote 1 SEM. $\quad * p<.05$. 
havior during the second postshift trial was replicated, and it was also shown that this ejaculatory effect did not last long, as measured in the subsequent postshift trials, and thus, could be considered as a transitory effect.

If the anxiolytic-like effect of ejaculation on defensive burying behavior (Fernández-Guasti et al., 1989; RodríguezManzo et al., 1999; Saldívar et al., 1991) and the different consequences of anxiolytic agents on the behavior of rats during the first and the second trials of reward downshift situations (Becker \& Flaherty, 1982; Flaherty, Grigson, et al., 1990; Flaherty, Grigson, \& Lind, 1990; Flaherty et al., 1996) are considered, the present results are consistent with an anxiolytic effect of ejaculation upon frustration. Still, we first need to assess other potential accounts of the present data.

First, the consummatory recovery (Experiments 2 and 3 ) and the decreased ambulatory (Experiment 2) and rearing (Experiment 3) behaviors of ejaculators, found in the second postshift trial, could be attributed just to an incremented thirst or lack of energy and to the apparent inactivity following copulation. This hypothesis, however, is inconsistent with the results of Experiment 1, in which the nonshifted animals in Group Ej4-4 did not show differences either in their goal-tracking time or in their ambulatory behavior - despite having had copulatory activityin comparison with the rats in Group 4-4, which were not exposed to females. Besides, Experiment 1's frustrated animals (i.e., Groups 32-4 and Ej32-4) did not differ among themselves in any of the dependent measures, despite the fact that the animals in one group had ejaculated twice before the reward shift, whereas the males in the other group had had no copulatory activity. In addition, in opposition to the idea of lower activity after copulation, Rodriguez-Manzo et al. (1999) found no differences between males achieving one ejaculation and controls unexposed to females in an ambulatory behavior test.

Discarding the tired thirsty rat explanation, it may be that contact with a receptive female or the copulatory activity itself (i.e., not ejaculation) causes the anxiolytic effect found in the second postshift trial in the cSNC situation. Although we did not test these alternative hypotheses, we were not totally oblivious to them either. Fernández-Guasti et al. (1989) had found that animals copulating for just five intromissions behaved similarly to those unexposed to females in the burying behavior test. Moreover, the male rats belonging to any of those two conditions (i.e., five intromissions and unexposed to females) presented significantly more cumulative burying behavior than did the animals in the ejaculatory group. Thus, taking into consideration that five intromissions and none at all presented the same lack of anxiolytic effects on the burying behavior test, we chose to use noncopulatory controls in the present study. Nevertheless, a five-intromission control remains to be tested in a cSNC situation, although it is expected to have no effect upon frustration.

Another potential account of the results obtained in Experiments 2 and 3 involves memory interference. Presumably, animals must compare the postshift reward with the memory of the preshift reward in order to show a contrast effect (Spear, 1967). In accordance with this hypothesis, a loss of the contrast effect has been found over 42- and 68-day retention intervals between the preshift and the postshift phases (Flaherty, 1996). On that basis, the consummatory recovery of ejaculators in the second postshift trial in Experiment 2 could be interpreted as a deficient memory of the preshift solution, due to interference. This means that those males that ejaculated had gone through an event (i.e., copulation) that could have interfered with memories of their past experience with the higher reinforcer. Therefore, when they faced the 4\% solution, they drank more of it than did the controls that had a better (i.e., not interfered with) recall of the $32 \%$ solution. However, this memory interference ad hoc hypothesis for the present data cannot account for the lack of differences between the ejaculators and the nonejaculators in Experiment 1.

In summary, considering that we are able to discard other potential accounts of the present data, the results of this study are consistent with the idea that two distinct sequential behavioral/physiological processes take place in animals facing a reward downshift and are also consistent with both Amsel's (1958, 1992) and Flaherty's (1996) theories of contrast phenomena. In short, we have found an effect of ejaculation upon frustration in the second postshift trial, but not in the first one, a finding that is similar to what has been found when diverse anxiolytic agents were used. In addition, we have shown that this replicated ejaculatory effect was transitory - that is, it affected the males' consummatory behavior only during the immediate subsequent postshift trial, but not during the next trials - which is again a result similar to those that have been found in contrast studies in which anxiolytic drugs have been used. Hence, it seems valid to affirm that we have extended ejaculatory antianxiety effects to a stressful situation without presentation of nociceptive stimuli such as cSNC.

Fernández-Guasti and Saldívar (1990) have reported that the reduction in anxiety after ejaculation, which is found in the burying behavior test, was mediated via an increment in GABAergic transmission. Besides, drug data suggest that anxiolytics may hasten the recovery from cSNC via a GABAergic mechanism (Flaherty, 1996). Therefore, further experiments in which pharmacological methods are used to modify anxiety states, such as selective benzodiazepine agonists, inverse agonists, and antagonists (Fernández-Guasti, Roldán-Roldán, \& Saldívar, 1990) would need to be undertaken in order to confirm the brain mechanisms that mediate the anxiolytic-like effect of ejaculation upon frustration found in the present study.

\section{REFERENCES}

Amsel, A. (1958). The role of frustrative nonreward in noncontinuous reward situations. Psychological Bulletin, 55,102-119.

Amsel, A. (1992). Frustration theory: An analysis of dispositional learning and memory. New York: Cambridge University Press.

BECKER, H. C. (1986). Comparison of the effects of the benzodiazepine midazolam and three serotonin antagonists on a consummatory con- 
flict paradigm. Pharmacology Biochemistry \& Behavior, 24, 10571064.

BeCKer, H. C., \& Flaherty, C. (1982). Influence of ethanol on contrast in consummatory behavior. Psychopharmacology, 77, 253-258.

Coe, C. L., Stanton, M. E., \& Levine, S. (1983). Adrenal responses to reinforcement and extinction: Role of expectancy versus instrumental responding. Behavioral Neuroscience, 97, 654-657.

DALY, H. B. (1974). Reinforcing properties of escape from frustration. In G. H. Bower (Ed.), The psychology of learning and motivation (Vol. 7, pp. 187-232). New York: Academic Press.

FernándeZ-Guasti, A., Roldán-Roldán, G., \& Saldívar, A. (1989). Reduction in anxiety after ejaculation in the rat. Behavioural Brain Research, 32, 23-29.

FernándeZ-Guasti, A., Roldán-Roldán, G., \& Saldívar, A. (1990). Pharmacological manipulation of anxiety and male rat sexual behavior. Pharmacology Biochemistry \& Behavior, 35, 263-267.

Fernández-Guasti, A., \& Saldívar, A. (1990). Participation of the GABA-benzodiazepine system in the inhibition of defensive burying produced by ejaculation. Behavioral Pharmacology, 1, 429-436.

FlaherTy, C. F. (1996). Incentive relativity. New York: Cambridge University Press.

Flaherty, C. F., Becker, H. C., \& Pohorecky, L. (1985). Correlation of corticosterone elevation and negative contrast varies as a function of postshift day. Animal Learning \& Behavior, 13, 309-314.

Flaherty, C. F., Blitzer, R., \& Collier, G. H. (1978). Open-field behaviors elicited by reward reduction. American Journal of Psychology, 91, 429-443.

Flaherty, C. F., Clarke, S., \& Coppotelli, C. (1996). Lack of tolerance to contrast-reducing actions of chlordiazepoxide with repeated reward reductions. Physiology \& Behavior, 60, 645-652.

Flaherty, C. F., Greenwood, A., Martin, J., \& Leszczuk, M. (1998). Relationship of negative contrast to animal models of fear and anxiety. Animal Learning \& Behavior, 26, 397-407.

Flaherty, C. F., Grigson, P. S., Demitrikopoulos, M. K., Weaver, M. S., Krauss, K. L., \& Rowan, G. A. (1990). Effect of serotoninergic drugs on negative contrast in consummatory behavior. Pharmacology Biochemistry \& Behavior, 36, 799-806.

Flaherty, C. F., Grigson, P. S., \& Lind, S. (1990). Chlordiazepoxide and the moderation of the initial response to reward reduction. Quarterly Journal of Experimental Psychology, 42B, 87-105.

Flaherty, C. F., Grigson, P. S., \& Rowan, G. A. (1986). Chlordiazepoxide and the determinants of contrast. Animal Learning \& Behavior, 14, 315-321.

Freidin, E., \& Mustaca, A. E. (2004). Frustration and sexual behavior in male rats. Learning \& Behavior, 32, 311-320.
GraY, J. A. (1977). Drug effects on fear and frustration: Possible limbic site of action of minor tranquilizers. In L. L. Iversen, S. D. Iversen, \& S. H. Snyder (Eds.), Handbook of psychopharmacology: Vol. 8. Drugs, neurotransmitters, and behavior (pp. 433-529). New York: Plenum.

GraY, J. A. (1982). The neuropsychology of anxiety: An enquiry into the function of the septo-hippocampal system. Oxford: Oxford University Press.

GraY, J. A. (1985). A whole and its parts: Behavior, the brain, cognition and emotion. Bulletin of the British Psychological Society, 38, 99-112.

Mitchell, C., \& Flaherty, C. (1998). Temporal dynamics of corticosterone elevation in successive negative contrast. Physiology \& Behavior, 64, 287-292.

Mustaca, A. E., Bentosela, M., \& Papini, M. R. (2000). Consummatory successive negative contrast in mice. Learning \& Motivation, 31, 272-282.

Mustaca, A. E. Freidin, E., \& Papini, M. R. (2002). Extinction of consummatory behavior in rats. International Journal of Comparative Psychology, 1, 1-10.

Mustaca, A. E., \& Martelli, M. I. (2000). Máxima discrepancia en el contraste consumatorio. VII Jornadas de psicología, Faculty of Psychology, University of Buenos Aires.

PapinI, M. R., \& Dudley, R. T. (1997). Consequences of surprising reward omissions. Review of General Psychology, 3, 275-285.

Pecoraro, N. C., Timberlake, W. D., \& Tinsley, M. (1999). Incentive downshifts evoke search repertoires in rats. Journal of Experimental Psychology: Animal Behavior Processes, 25, 153-167.

Pellegrini, S., \& Mustaca, A. (2000). Consummatory successive negative contrast with solid food. Learning \& Motivation, 31, 200-209.

Rodríguez-Manzo, G., López-Rubalcava, C., \& FernándezGuAsTI, A. (1999). Anxiolytic-like effect of ejaculation under various sexual behavior conditions in the male rat. Physiology \& Behavior, 67, 651-657.

Saldívar, A., Ríos, C., \& Fernández-Guasti, A. (1991). Differential role of serotonin and noradrenaline on anxiety reduction after ejaculation in the rat. Pharmacology Biochemistry \& Behavior, 38, 807-812.

Spear, N. E. (1967). Retention of reinforcement magnitude. Psychological Review, 74, 216-234.

Treit, D., Pinel, J. P. J., \& Fibiger, H. C. (1981). Conditioned defensive burying: A new paradigm for the study of anxiolytic agents. Pharmacology Biochemistry \& Behavior, 15, 619-626.

(Manuscript received July 7, 2004; revision accepted for publication October 4, 2004.) 